\title{
Anesthesiologist's Perspective on the Standardization of Clinical Terminology in Electronic Health Records
}

\section{Kyoung Ok Kim}

Department of Anesthesiology and Pain Medicine, Dongguk University Ilsan Hospital, Goyang, Korea

Over the past decades, paper medical records have been replaced by Electronic Health Records (EHRs) in many hospitals. EHRs are expected to improve patient care and safety by allowing clinicians to share important health information that could influence patients' outcomes [1]. From a research perspective, EHRs provide a tool for capturing, sharing, and aggregating data for analysis in multi-center clinical observational studies. However, variability in methods of recording and capturing data constitutes a major barrier to sharing and integrating data between institutions.

Clinicians often use different terms to describe the same thing or the same term to describe different things, and anesthesiologists are no exception. The growth of electronically stored records has been accompanied by a need for standardization to improve data quality and interchangeability $[2,3]$. Structured documentation using standardized clinical termi-

Submitted: April 22, 2021

Revised: July 12, 2021

Accepted: July 26, 2021

\section{Corresponding Author}

Kyoung Ok Kim

Department of Anaesthesiology and Pain Medicine, Dongguk University Ilsan Hospital, 27, Dongguk-ro, Ilsandong-gu, Goyang 10326, Korea. Tel: +82-31-961-7874,E-mail: pissces@dumc.or.kr (https://orcid.org/0000-0001-7509-3668)

This is an Open Access article distributed under the terms of the Creative Commons Attribution Non-Commercial License (http://creativecommons.org/licenses/by$\mathrm{nc} / 4.0 /$ ) which permits unrestricted non-commercial use, distribution, and reproduction in any medium, provided the original work is properly cited.

(C) 2021 The Korean Society of Medical Informatics nology can resolve the issue of variability by providing clinicians with a common language. Regardless of the quantity of data recorded in the EHR, if the proper information cannot be retrieved, it is useless and a waste of resources. Nonetheless, the standardization of health data is in its infancy, and this issue has received relatively little attention from clinicians-and, again, anesthesiologists are no exception.

Anesthesia-derived data, which are straightforwardly constrained in terms of items and structure, have distinct advantages over the documentation produced in many other medical specialties. For example, outpatient visit documents or surgical records may include a number of variables that cannot be easily structured into a standardized format, whereas a narrative description is suitable for a broad range of possible issues. However, most data gathered during an anesthesia case are commonly present across anesthesia encounters, and some can be predefined for categorization into one of several groups to fit into a structured format. Furthermore, the charting elements themselves can be limited to a few possible options (e.g., patient classification according to the American Society of Anesthesiologists [ASA] or Cormack grade). A well-structured anesthesia document can accurately capture a patient's anesthesia experience in a concise format, allowing anesthesiologists to retrieve important information regarding patient care and safety.

Multi-center clinical observational studies using multiple data sources are becoming increasingly common. A multicenter study can provide network-wide results by conducting the same protocols at the participating organizations over a short period and then combining the summarized results for 
analysis. This approach improves the statistical power, and the increased sample size helps to reveal more significant relationships. New major amendments to data privacy laws have been introduced in Korea, and pseudonymized data can be processed without the subject's consent if the intended use is within the boundaries of preparing statistics and scientific research [4]. When conducting multi-center observational studies, data can be used in more flexible ways according to the new law. Despite the introduction of EHRs, which enable data-sharing and integration, multi-center clinical observational studies are still difficult to run. Inhomogeneity in the data constitutes a major obstacle to integrating data among the research partners. Therefore, standardization of EHR structure, content, and vocabulary should be considered.

Although anesthesiology data has characteristics suitable for standardization, standardization work is lagging behind in the anesthesiology field. However, there is no need to start from the beginning, since several prominent standard development organizations already exist. For example, Systematized Nomenclature of Medicine Clinical Terms (SNOMED CT) consists of clinical terminology code sets developed by SNOMED International [5]. Fortunately, the Ministry of Health and Welfare designated the Korea Health Information Service as the SNOMED CT national release center, and Korea became the 39th official member of SNOMED International in August 2020. All domestic medical institutions can use the system freely without individual subscriptions to SNOMED International or additional fees [6]. SNOMED CT provides well-organized terminology for diagnosis, conditions, and outcomes, but not for vocabularies related to anesthesiology. Some of the anesthesia-related terminology in SNOMED CT is semantically ambiguous, some terms are duplicated, and some essential terms are not included or need improvement.

Anesthesia information is gathered during preoperative, intraoperative, and postoperative phases. Preoperative information, including the Mallampati classification from an airway examination, the ASA physical status classification, and concurrent disease, should be included in the structured anesthesia documentation. The type of anesthesia, anesthetic agents, airway condition, and postoperative complications (e.g., postoperative nausea and vomiting) may help with patient management in the future. Critical information for patient safety, such as the Cormack grade for difficult airway cases, could later be used to build an airway registry without much effort, ensuring patient safety.

Clearly, the implementation of standardized clinical terminology in EHRs will improve patient safety and the quality of clinical research. To achieve this goal, first of all, anesthesiologists should actively participate in the standardization of anesthesia vocabularies, which contain terms that are mainly used only in the field of anesthesia. Second, the anesthesia community must agree on the items necessary for anesthesiarelated documentation. Last but not least, efforts need to be made to increase awareness of the importance of standardized terminology among individual anesthesiologists and to develop a user-friendly EHR structure that allows essential information to be filled out accurately. If we wish to utilize EHRs for patient safety and research, anesthesiologists need to take the initiative in the standardization of vocabularies.

\section{Conflict of Interest}

No potential conflict of interest relevant to this article was reported.

\section{ORCID}

Kyoung Ok Kim (http://orcid.org/0000-0001-7509-3668)

\section{References}

1. Elevitch FR. SNOMED CT: electronic health record enhances anesthesia patient safety. AANA J 2005;73(5):361-6.

2. Boyce RD, Ryan PB, Noren GN, Schuemie MJ, Reich C, Duke J, et al. Bridging islands of information to establish an integrated knowledge base of drugs and health outcomes of interest. Drug Saf 2014;37(8):557-67.

3. Yoon D, Ahn EK, Park MY, Cho SY, Ryan P, Schuemie MJ, et al. Conversion and data quality assessment of electronic health record data at a Korean tertiary teaching hospital to a common data model for distributed network research. Healthc Inform Res 2016;22(1):54-8.

4. Personal Information Protection Commission. Personal Information Protection Act (General Law) [Internet]. Seoul, Korea: Personal Information Protection Commission; c2021 [cited at 2021 Sep 30]. Available from: https://www.privacy.go.kr/eng/laws_view. do? nttId=8186\&imgNo=3.

5. SNOMED International [Internet]. London, UK: SNOMED International; c2021 [cited at 2021 Sep 30]. Available from: https://www.snomed.org.

6. Korea Health Information System. SNOMED CT NRC [Internet]. Seoul, Korea: Korea Health Information System; c2021 [cited at 2021 Sep 30]. Available from: https://www.k-his.or.kr/menu.es?mid=a20203010000. 